Service social

\title{
La recherche qualitative au 54e congrès de l'ACFAS
}

\section{Jean-Pierre Deslauriers}

Volume 35, numéro 1-2, 1986

Recherche - Action - Évaluation

URI : https://id.erudit.org/iderudit/706303ar

DOI : https://doi.org/10.7202/706303ar

Aller au sommaire du numéro

Éditeur(s)

École de service social de l'Université Laval

ISSN

1708-1734 (numérique)

Découvrir la revue

Citer cet article

Deslauriers, J.-P. (1986). La recherche qualitative au 54e congrès de l'ACFAS

Service social, 35(1-2), 242-245. https://doi.org/10.7202/706303ar d'utilisation que vous pouvez consulter en ligne.

https://apropos.erudit.org/fr/usagers/politique-dutilisation/ 


\section{La recherche qualitative au 54e congrès de l'ACFAS}

\section{Jean-Pierre Deslauriers}

La recherche qualitative a été bien servie lors du $54^{\mathrm{e}}$ congrès annuel de l'Association canadienne-française pour l'avancement des sciences (ACFAS), tenu à l'Université de Montréal du 16 au 20 mai 1986. En effet, la section "Éducation » avait organisé un atelier thématique sur la méthodologie qualitative, et la section "Sociologie" avait fait de même le même jour et à la même heure ! Pris pour choisir, il m'a semblé que le premier était plus intéressant. De plus, l'Association québécoise pour la recherche qualitative (A.R.Q.) avait organisé, le 15 mai, son cinquième colloque sous le thème: "La science peut-elle apprendre des acteurs sociaux?".

Le terme de recherche qualitative peut regrouper toutes sortes d'approches mais, à Montréal, on discuta surtout de recherche-action et d'histoire de vie.

André Morin (Université de Montréal) présenta une communication visant à définir un peu plus précisément la recherche-action. À la suite de ses travaux antérieurs, il a développé une grille formée de cinq éléments (le contrat, la participation, le discours, le changement, l'acteur) lui permettant d'analyser et de classer les projets de rechercheaction. Encore une fois (et ce n'est pas la dernière!), la question rebondit : la recherche-action constitue-t-elle ou non une méthode de recherche scientifique? Produit-elle des connaissances sûres? À ce sujet, la présentation d'André Morin suscitait des questions auxquelles Guy Le Boterf (Paris) allait essayer de répondre le lendemain, au colloque de l'A.R.Q.

Les deux auteurs en proposaient une définition différente. Morin met l'accent sur le changement, "La recherche-action vise un changement par la transformation réciproque de l'action et du discours, i.e. d'une action individuelle à une pratique collective efficace 
et incitatrice, d'un discours spontané à un dialogue éclairé et engagé ". Le Boterf, quant à lui, insiste sur le processus de résolution de problèmes, i.e. "La recherche-action est le processus dans lequel les acteurs participent à l'analyse et à la résolution de leurs problèmes". Toutefois, malgré leurs différences, les deux définitions illustrent ce qui sépare la recherche-action de la recherche plus conventionnelle.

Comme le soulignait Le Boterf, le savoir produit par la rechercheaction est plus localisé, plus lié à l'action et à la solution d'un problème concret ; en ce sens, il est moins généralisable et ne répond pas toujours aux critères positivistes de la science. Peut-être serait-il préférable de parler d'étude plutôt que de recherche. Ensuite, les conditions de production sont différentes : la recherche se protège traditionnellement contre l'action alors que la recherche-action l'appelle et la sollicite. Différence de logique aussi : l'une vise surtout la transformation du savoir alors que l'autre est à l'affût du savoir pratique orienté vers l'action. De ce point de vue, la recherche-action, tout comme les autres approches de recherche qualitative, donne accès au point de vue des acteurs, aux aspects plus microscopiques de la réalité, au spontané, à l'informel. L'action peut donc aussi s'avérer un moyen de recherche.

Comme d'autres communications l'ont démontré, entre autres celles de Jean-Marie Bouchard et de Nicole Vézina (Université du Québec à Montréal), la recherche-action ne produit pas automatiquement des connaissances nouvelles: souvent elle valide la connaissance inhérente à l'action. Le chercheur codifie le savoir informel en traduisant l'expérience en écrit. Un renversement de perspectives serait donc en train de s'opérer en sciences humaines : au lieu de guider les masses par son savoir, l'intellectuel/chercheur se met à l'écoute de la réalité et s'y associe. Cependant, cette position plus humble, et probablement plus réaliste aussi, cache une grande faiblesse: si la recherche-action ne fait que reproduire la réalité et ce que donne le sens commun, ne manque-t-elle pas d'une méthodologie qui nous permette de passer du connu à l'inconnu, du manifeste au théorique? La connaissance se base sur la réalité, mais aussi sur ce qui ne se voit pas. Enfin, c'est une question de méthode qu'il faudra bien éclaircir.

II faut aussi distinguer "la méthode» de recherche de son "orientation». Rien n'assure que la recherche-action, ni aucune autre méthode d'ailleurs, soit automatiquement orientée vers le changement social; on sait qu'elle peut également manifester des tendances technocratiques. D'autre part, il est tout aussi exagéré de penser que la recherche traditionnelle est absolument étrangère aux préoccupations contemporaines: des travaux économétriques serrés portant sur la distribution inégale des revenus présentent un potentiel évident de 
critique radicale de la société. Ce n'est pas qu'une question de méthode, mais surtout d'objectifs et de préoccupations du chercheur.

Il m'a semblé que la recherche appliquée, voire applicable, exerçait un grand attrait pour les promoteurs de la recherche qualitative. Ils/elles ont bien raison : le chercheur dans sa tour d'ivoire, en plus de présenter une mauvaise image, est un mythe. La recherche sociale est partie prenante de son époque: le chercheur remplit une fonction précise et ses travaux ne sont pas sans influence; dans ces conditions, aussi bien essayer de répondre à des besoins concrets. De ce point de vue, comme l'a souligné avec pertinence Hector Ouellet (Centre de recherche sur les services communautaires, Université Laval), il n'est pas démontré qu'accorder une plus grande importance à la recherche appliquée affaiblira la recherche fondamentale. De fait, les deux peuvent en tirer profit. Toutefois, il ne faut pas jeter le bébé avec l'eau du bain : la rentabilité immédiate de la recherche, l'application à tout prix, ne constitue pas un progrès en soi mais peut s'avérer un recul. De plus, les disciplines les plus appliquées se développent, la plupart du temps, grâce aux apports d'autres disciplines plus théoriques. En ces temps difficiles où triomphent le conservatisme et les expédients, il y a plus que jamais place pour les études qui réorientent et reconceptualisent ; rien n'empêche la recherche-action d'y contribuer à sa façon.

L'autre grand thème, celui des histoires de vie, a été abordé dans trois communications intéressantes. Tout d'abord, Adèle Chené (Université de Montréal) présenta les résultats de l'analyse d'une quarantaine de courts récits produits par des étudiant(e)s adultes; à partir de ces autobiographies rédigées à la fin de leurs études, il a été possible de retracer les étapes communes (décision, acquisition, sanction) et de démontrer que le récit de formation peut aussi être un moyen de réappropriation de son identité personnelle ou professionnelle.

Jeanne-Marie Gingras (Université de Montréal) a ensuite traité de I'histoire de vie comme processus de création : elle prétend que le récit et la création présentent des cheminements semblables et remplissent tous deux des fonctions d'intégration et de transformation de l'expérience. Cette présentation soulevait les questions suivantes : estce que l'histoire de vie, fût-elle celle de l'acte créatif lui-même, exprime seulement la personne ou son insertion dans la société ? la création estelle un processus individuel pur ou l'expression individuelle d'un processus social? Comme s'interrogeait Bertaux, les histoires de vie constituent-elles des récits de vie ou des récits de pratique? Comme il arrive souvent à l'ACFAS, le temps a manqué pour approfondir cette question. 
Jean-Pierre Theil (Université de Montréal) mène, depuis quelque temps, une passionnante étude sur le processus d'apprentissage des autodidactes : à partir de récits de vie, il a essayé de reconstruire leur parcours, et il en est ressorti des résultats fascinants. Tout d'abord, ils ne possèdent pas de cadre de référence que fournit habituellement la discipline: pour pallier à cette faiblesse, ils accordent une grande importance au dialogue avec les autres pour contrôler leurs informations. Ils rechercheront donc les informateurs et informatrices qui les aideront à sélectionner les renseignements et évaluer leurs sources. Grosso modo, l'autodidacte rencontre les mêmes problèmes que le chercheur (informations sûres, instrument de cueillette de données adéquat, analyse précise) mais, le plus étonnant est qu'il les solutionne de la même façon par la triangulation, la comparaison, la rationalisation! Quelle belle intuition: le processus de recherche ne serait-il que la systématisation du sens commun, une procédure savante qui se rencontre à l'état brut dans la vie quotidienne? La recherche de l'autodidacte serait la recherche qualitative en action.

Le 15 mai, I'A.R.Q. avait invité Alberto Cambrioso (Massachusetts Institute of Technology, Cambridge) à présenter ses réflexions sur le processus de formation des connaissances scientifiques. Très souvent, la science sociale prend les sciences naturelles comme modèle et tente d'utiliser les mêmes moyens pour élaborer les connaissances; ainsi, le laboratoire et l'expérimentation sont privilégiés. Cependant, après avoir observé les naturalistes, il apparaît que leur pratique, loin d'être aussi rationnelle qu'on prétend, est aussi empreinte de pensée magique et de sens commun! Le drame des sciences sociales est peut-être qu'elles essaient d'imiter les sciences naturelles dans leur prétention plutôt que dans leur réalité. Quoi qu'il en soit, étudier l'épistémologie spontanée des scientifiques peut s'avérer une piste de recherche très féconde.

La journée s'est terminée avec le rapport du président et du trésorier de I'A.R.Q. Il appert que le réseau va en s'élargissant et que nous pouvons compter environ trois cents personnes intéressées par la recherche-action ou la recherche qualitative en général. Les finances vont bien et on essaiera d'autofinancer les activités, dont la publication d'un bulletin. On projette aussi d'organiser deux colloques par année, un à l'automne et un autre qui coönciderait avec le congrès de l'ACFAS. Bref, l'avenir de la recherche qualitative apparaît prometteur. 